\title{
KOMPETENZENTWICKLUNG FÜR ÜBERSETZER UND DOLMETSCHER- AM BEISPIEL DES INSTITUTS FÜR TRANSLATIONSWISSENSCHAFT DER UNIVERSITÄT MARIBOR
}

\author{
Vlasta Kučiš
}

Abteilung für Translationswissenschaft, Philosophische Fakultät, Universität Maribor, Maribor, Slowenien

Zusammenfassung

Der Beitrag ist ein erster Versuch, den neu gegründeten Studiengang Übersetzen und Dolmetschen an der Philosophischen Fakultät der Universität Maribor zu analysieren und seine Hauptcharakteristika zu beleuchten. Es wird versucht aufzuzeigen, dass der Aufbau des Studiums auf translatorischer Kompetenzentwicklung mit theoretischen und didaktischen Ansätzen basiert. Die Integrationspolitik der EU setzt auf qualifizierte Übersetzer- und DolmetscherInnen und der Übersetzerberuf strebt nach unifizierten Ausbildungskriterien. Theoretisch-wissenschaftliche Postulate untermauern die dargestellte Ausbildungsstrategie im Kontext der Harmonisierung und Optimierung der europäischen Hochschul-Studiengänge und -Curricula für angehende TranslatorInnen.

Schlüsselwörter

Translationswissenschaft; Ausbildungsprogramm; Übersetzer

\section{Einleitung}

Im „Europäischen Jahr des interkulturellen Dialogs 2008" ist es für die slowenische und damit europäische Translationswissenschaft nicht nur sinnvoll, sondern auch notwendig, sich über die translatorische und interkulturelle Ausbildung Gedanken zu machen. Migration, Mobilität und sprachlich-kulturelle Vielfalt prägen Europa seit Jahrhunderten und Mehrsprachigkeit und Plurikulturalität gehören zu den Hauptcharakteristika des alten Kontinents. In zahlreichen europäischen Ländern hat die translatorische Ausbildung eine lange Tradition, während sich in anderen Ländern entsprechende Studiengänge erst im Entstehungsprozess bzw. in der Aufbauphase befinden, wie z.B. an der Universität Maribor, oder sogar noch ganz fehlen. Die Europäische Kommission bzw. Generaldirektion Übersetzung (DGT) /1/ übernimmt bei der Ausbildung von TranslatorInnen eine aktive Rolle, indem sie professionelle als auch finanzielle Hilfestellung leistet, unter anderem mit dem Ziel, eine Harmonisierung der universitären Studiengänge und Curricula zu gewährleisten. Für eine Intensivierung von Optimierung und Kompatibilität der Ausbildungsgänge für Übersetzer und Dolmetscher und die entsprechende Sensibilisierung von Lehrkräften und Studierenden gibt es mehrere relevante Gründe:

- Zunehmende Globalisierungsprozesse, Marktentwicklung und technisch bedingte Fortschritte stellen quantitativ und qualita- 
tiv neue Anforderungen an die TranslatorInnen

- Die Integrationspolitik der EU setzt auf qualifizierte Übersetzer- und DolmetscherInnen, die u.a. auch über die Einstellungskriterien und - verfahren der europäischen Institutionen informiert sind

- Der nicht reglementierte Übersetzerberuf strebt nach unifizierten Exzellenz-Kriterien

- Arbeitsbedingungen und Einkommen der TranslatorInnen streben nach Aufwertung.

- Der Vielzahl der Ausbildungsprogramme sollte - im Zuge des Bologna-Prozesses - ein gemeinsames Minimum an translatorischen Kompetenzen zugrunde liegen.

Unter diesen Gesichtspunkten wird in den folgenden Kapiteln die Ausbildung der TranslatorInnen an der Universität Maribor analysiert und kommentiert, wobei der Entwicklung der translatorischen Kompetenz besondere Aufmerksamkeit zukommt und diese mit Hilfe der bestehenden theoretisch-wissenschaftlichen Konzepte der aktuellen Translationswissenschaft belegt wird.

\section{Retrospektive der TranslatorInnenausbil- dung an der Universität Maribor}

An der Universität Maribor wurde die Einführung eines Translationsstudiums bereits vor sechs Jahren intensiv in Betracht gezogen. Mit dem Ziel, den europäischen Tendenzen zu folgen und das Konzept von Mehrsprachigkeit und kultureller Vielfalt zu etablieren, ging man dann innerhalb der Lehrstühle für Germanistik und Anglistik an die Einrichtung eines Studiengangs für Übersetzen und Dolmetschen. Beide Lehrstühle waren mit ihren methodischdidaktisch konzipierten Lehrplänen primär pädagogisch, d.h. auf künftige Lehramtskandidaten ausgerichtet, und der neue ÜbersetzerStudiengang wurde als nicht-pädagogisch konzipiertes Programm teilweise in das bestehende Studienprogramm inkorporiert. Das sprachund literaturwissenschaftliche Konzept des Curriculums wurde übernommen und durch einige translationsrelevante Seminare und Übungen bereichert, was aber, wie sich herausstellte, für die notwendige Differenzierung und, damit verbunden, die spezifische Kompetenzentwicklung von potenziellen Übersetzerund DolmetscherInnen nicht ausreichte. Zwar war man bemüht, die Ausbildung der TranslatorInnen professionell und in Theorie und Praxis auch kompetent zu gestalten, doch Studenten und Lehrkräften war mit dieser Regelung nicht gedient. Akzeptabel war sie vielmehr lediglich als möglichst kurze Übergangsphase. Aus heutiger Sicht kann dieses hybride Studium vielleicht als Pionierarbeit eingestuft werden, denn mit entsprechenden Studienprogrammen in anderen europäischen Ländern war es nicht kompatibel. Innerhalb des stark linguistisch und literarisch belasteten Curriculums konnten die theoretischen und praktischen Aspekte der Translationswissenschaft nur ansatzweise entwickelt und artikuliert werden, was letztendlich dann zur Gründung des Instituts für Übersetzen und Dolmetschen an der Philosophischen Fakultät führte.

\section{Struktur des Translatorikstudiums- aktu- eller Stand}

Um die Lücke in der translatorischen Ausbildung an der Universität in Maribor $\mathrm{zu}$ schließen, wurde im Geiste der europäischen Kompetenzprofilentwicklung für mehrsprachige und multikulturelle Kommunikationsexperten im Jahre 2007 die Idee geboren, im Rahmen der Philosophischen Fakultät mit der Gründung des Instituts für Übersetzen und Dolmetschen einen neuen Studiengang einzurichten. Die Ausbildung der ÜbersetzerInnen und DolmetscherInnen an diesem Institut dauert, mit Beginn des Wintersemesters 2008/09, insgesamt zehn Semester (sechs Semester BAProgramm und vier Semester MA-Studium). Da das Curriculum von internationalen Translatologie-Experten begutachtet wurde, ist es mit bereits anerkannten europäischen universitären Studienprogrammen durchaus kompatibel. Nach sechs Semestern kann ein BA-Diplom als Sprachmittler für Deutsch und/oder Englisch und/oder Ungarisch erworben werden.

Unter den Translationswissenschaftlern als auch Berufsübersetzern herrscht ein Konsensus darüber, dass der BA-Studiengang keine Fachübersetzer und erst recht nicht Dol- 
metscher hervorbringen kann. Eine übersetzerische bzw. dolmetscherische Abschlussqualifikation, die vom internationalen Arbeitsmarkt gefragt wird, kann man an der Universität Maribor erst nach einem MA-Studium erwerben, das insgesamt 10 Semester dauert und mit einem Magister-Diplom für Übersetzen oder Dolmetschen endet.

Im Vordergrund des BA-Studienprogramms stehen praktische Übungen, wobei man sich mit Themen und Texten aus dem Alltagsbereich beschäftigt.

In den ersten zwei Semestern wird die Aufmerksamkeit der mündlichen und schriftlichen Kompetenzentwicklung gewidmet. Deren Zweck ist es, Informationen zu empfangen und $\mathrm{zu}$ vermitteln und parallel eine kompakte Basis für das Übersetzen und Dolmetschen zu bilden. Für den Fremdsprachenerwerb ist sowohl die Kommunikation von Bedeutung, als auch das Verständnis der Kultur, in deren Rahmen die Kommunikation stattfindet. Deswegen wird die Kommunikationskompetenz aus zwei verschiedenen Perspektiven betrachtet:

- als individuelle Kommunikation in der

Form des Verstehens und Produzierens von Informationen und

- als Entwicklung der Sprachgebrauchsfähigkeiten in der Form der Kompeten-

zentwicklung, die Sprache zu verstehen und produktive Sprachkenntnisse zu entwickeln. In diesem Kontext werden Texte aus den Bereichen Tourismus, Sport, Kultur, Wirtschaft und Politik bearbeitet, wobei sich die verbale Kommunikation in der Form der Rezeption und Produktion reflektiert. In der ersten Phase wird in die Muttersprache übersetzt, anschließend in die Fremdsprache, was für Studierende eine besondere Herausforderung bedeutet, da „Übersetzung - im weiteren Sinne - immer Kulturarbeit, in einem engeren Sinne Spracharbeit ist: Arbeit mit der anderen und an der eigenen Kultur, Arbeit mit und an der eigenen Sprache." /2/ Die Auswahl der Textsorten hängt auch von den LektorInnen ab, die bei der Erstellung ihrer Unterrichtsmaterialien freie Hand haben. Um den Studenten einen permanenten Einblick in ihre Leistungsqualität und Entwicklung der translatorischen Kompetenzen zu ermöglichen, erstellt jeder Student sein Por- tfolio /3/. Innerhalb der Ausbildung von TranslatorInnen werden auch Auslandsaufenthalte an verschiedenen Partneruniversitäten ermöglicht, die für die Studierenden von großer Bedeutung sind, was auch Ammann mit ihrer Behauptung untermauert: „Der Auslandsaufenthalt wird zur unabdingbaren Voraussetzung. Doch auch der Auslandsaufenthalt will vor- und nachbereitet sein. Die Nachbereitung umfasst auch die Reflexion der persönlichen Reaktion auf die fremde Kultur." /4/

Die organisatorischen Möglichkeiten des Instituts erlauben pro Jahr die Einschreibung von ca.120 Studenten, wobei ein kompetentes Unterrichtsprogramm geboten wird, das eine Fokussierung auf die grundlegenden Probleme der übersetzerischen und dolmetscherischen Praxis und Theorie gestattet.

Das didaktische Hauptziel des translatorischen Studiums ist demzufolge Entwicklung translatorischer Kompetenzen und damit verbundenen Förderung der sprachlichen und kulturellen Kenntnisse, um die Studierenden für die Mutter- als auch Fremdsprache zu sensibilisieren. Der Zusammenhang von Sprache und Kultur als auch Kommunikation als Informationstransfer und vor allem Translation/5/ als professionelles Problemlösen stehen im Vordergrund der Unterrichtskonzeption.

\section{Theoretische und didaktische Ansätze}

Bei der Entwicklung der didaktischen Konzeption für die Zwecke des Aufbaustudiums sind, wie schon angeführt, ein skopos-, handlungsund kommunikationsorientiertes Modell sowie psycholinguistische Modelle der Translation zur Basistheorie geworden. Die aus diesen Ansätzen abgeleiteten Perspektiven lassen das Beleuchten von für die Sprachmittlerausbildung relevanten Aspekten zu. Dabei handelt es sich nicht nur um die sprachlichen Faktoren der translatorischen Tätigkeit, sondern auch um Determinanten, die im außersprachlichen Bereich liegen und sich einem linguistischen $\mathrm{Zu}-$ griff entziehen.

Übersetzer und Dolmetscher machen als Sprachmittler und Kulturexperten die Kommunikation möglich und helfen durch gute Kenntnisse verschiedener Sprachen nicht nur 
sprachliche sondern auch kulturelle Barrieren zu überwinden. Demnach ist es eine sehr komplexe Aktivität, wenn man interkulturell bzw. Transkulturell /6/ tätig ist. Malgorzewicz behauptet: „Für ein reibungsloses Funktionieren des translatorischen Handelns als Sondersorte der interkulturellen Kommunikation muss der Übersetzer mit der Kultur der Ausgangs- und Zielsprachenbenutzer vertraut sein, um so die Verhaltensnormen und -konventionen der Angehörigen von verschiedenen Kulturen, denen seine Auftraggeber angehören, bemerken und beachten zu können. Seine Aufgabe besteht im Grunde darin, eine Brücke zwischen den jeweiligen Kulturkreisen zu bauen." /7/.

Das wesentliche Ziel einer jeden translatorischen Handlung ist Äquivalenzbeziehungen zwischen einem Ausgangstext und einem Zieltext aufzubauen. Dies erreicht der Translator dadurch, dass er den AS-Text einem kognitiven Transfer unterzieht. Den Aspekt der Kultur bei der Übersetzung betont auch Vermeer: „Der Übersetzer muss die Konventionen und Normen der Zielkultur und ihrer Vertextungsstrategien kennen. Er muss plurikulturell und plurilingual sein - wahrscheinlich zuerst plurikulturell: Sprachfehler verzeiht man leichter als weniger bewusste, aber anscheinend tiefer ansetzende soziale Verstöße." /8/ Geht man davon aus, dass Sprache und Kultur in der Kommunikation untrennbar miteinander verbunden sind, dann bilden die Sprach- und Kulturkompetenz in der Translationswissenschaft eine Symbiose. Auch Mary Snell-Hornby setzt sich in ihren Werken für die Erkenntnis ein, dass jeder Text in einem kulturellen Kontext eingebettet ist.

Die finnische Übersetzungswissenschaftlerin Holz-Mänttäri ersetzt den Begriff „Übersetzen“ mit dem Terminus "translatorisches Handeln" und versteht darunter eine Expertenhandlung. Beim Übersetzen müssen nicht nur linguistische, sondern auch kulturelle und soziologische Aspekte berücksichtigt werden. Der Aufgabengebiet des Translators wird damit erweitert. Neben der Vermittler- bekommt er auch auch eine Beraterfunktion.

Holz-Mänttäri spricht heute von Bewusstseinstransfer, der impliziert, dass der Translator die Inadäquatheit der gewohnten
Verhaltensweisen erkennen und einen professionellen Handlungsrahmen konstruieren kann, in dem er die relevanten Bedingungen der Zielsituation berücksichtigt $/ 9 /$.

Die AS-Textwelt wird durch Projektion auf die Zielsprache und Zielkultur in die kognitiven Denkschemata, in die kommunikativen Handlungskategorien des ZS-Adressaten und schließlich in seine Diskurswelt umprofiliert und damit in die Zielsprache überführt und ausdrückbar gemacht.

Dies kann unter der Voraussetzung geschehen, dass die vertretenen Kulturen der Kommunikationspartner, ihr Welt- und Diskurswissen, aktuelle Systeme, Strategien, Erfahrungen und Erlebnisse kompatibel sind bzw. kompatibel gemacht werden können. Diejenigen Inhalte und Schlüsselbegriffe der Kultur, die als inkompatibel und von dem Übersetzer als translatorisch relevant ausgewiesen werden, unterliegen dem Divergenztransfer. Gibt es etwa in der Ausgangssprache ein Wort und kein entsprechendes in der Zielsprache, so spricht Koller von „echten Lücken“ /10/. Für ÜbersetzerInnen sind jedoch echte Lücken nur "vorläufige“" Lücken /11/, da die Aufgabe des Transaltors darin besteht, dieses Problem auf eine andere Weise zu lösen. Dabei handelt es sich meistens um kulturspezifische Andersartigkeiten. In der einschlägigen wissenschaftlichen Literatur wird daher $\mathrm{zu}$ Recht betont, dass Translation »nicht nur ein sprachlicher, sondern immer auch ein kultureller Transfer ist« /12/. Für den Übersetzer heißt das: er muss sowohl die Ausgangs- als auch die Zielkultur kennen, weil sprachliche Tätigkeit in hohem Maße immer auch kulturelle Tätigkeit ist vgl. /13/. Das Problem wird besonders deutlich, wenn Texte übersetzt werden, in denen sich die kulturbedingten und textsortenspezifischen Konventionen von Ausgangs- und Zielsprache stark voneinander unterscheiden.

Da ist dann den zielsprachigen Konventionen Rechnung zu tragen, um eine gelungene interbzw. transkulturelle Kommunikation $\mathrm{zu}$ gewährleisten. Der Übersetzer muss von der Textfunktion ausgehen, denn der übersetzte 
Text soll und muss in der Zielsprache als Teil der Zielkultur funktionieren und adressatengebunden sein.

Die dargestellten theoretischen Konzeptionen setzen für die TranslatorInnenausbildung im Rahmen des BA-Studiums (Sprachmittler) und im MA-Programm (Übersetzer und Dolmetscher) folgende Lernziele voraus:

- Sensibilisierung für ein zweckorientiertes und kulturbedingtes Übersetzen

- Vermittlung von sprachlichen und kulturellen Grundlagen in der Mutter- und Fremdsprache; Erwerb von rezeptiver und produktiver Kompetenz

- Bewusstmachung, Wahrnehmung und Evaluierung von kulturellen Phänomenen

- Terminologiearbeit

- Verbesserung der kontrastiven Textkompetenz

- Systematisierung von kognitiven Bedingtheit von Translation

- Erwerb interkultureller Transferkompetenz

- Erweiterung des Fachwissens durch Übersetzung von Fachtexten

- $\quad$ Computergestütztes Übersetzen

\section{Translatorische Kompetenz}

Unter Kompetenz versteht man die Gesamtheit der Fähig- und Fertigkeiten, Kenntnissen und Verhaltensweisen, die für die Erledigung einer konkreten Aufgabe unter bestimmten Umständen erforderlich sind. Translatorische Kompetenz ist jene Fähigkeit, die den Übersetzungsvorgang bzw. das translatorische Handeln ermöglicht und über das allgemeine Kommunikationsvermögen des Translators einerseits sowie über bloße Fremdsprachenkenntnisse andererseits hinausgeht. Translatorisches Handeln bedeutet die Produktion eines Textes als Informationsangebot in der Zielsprache über einen Text, der als Informationsangebot in der Ausgangssprache vorliegt, unter Berücksichtigung des intendierten Rezipienten /14/. Von dem Konsumenten des Übersetzungsproduktes gehen auch Hönig/Kußmaul /15/ aus, für welche die übersetzerische Kompetenz durch die Wirkung, die der Translator mit seinem ZSText beim Adressaten erzielt, gekennzeichnet ist. Auch Vermeer fordert translatorische Kom- petenz, die über den lingustischen Rahmen hinausgeht, indem er fordert: „Die Bikulturalität des Translators erst vermag zwischen Angehörigen zweier sich sonst nicht oder nicht hinreichend verständlicher Kulturen zu vermitteln." /16/

Für den Transfer von der Ausgangssprache in die Zielsprache muss der Übersetzer allein wissen, auf welche Textsegmente er welche Übersetzungsmethoden und -techniken anwenden muss, wie z.B. wörtliche Übersetzung, nichtwörtliche Übersetzung, paraphrasierende Übersetzung, Auslassung bestimmter Elemente etc., um zu einem pragmatischen oder äquivalenten Gleichgewicht zwischen Ausgangs- und Zieltext zu gelangen.

Im Hinblick auf die dargestellten theoretischwissenschaftlichen Postulate lässt sich die translatorische Kompetenz in vier Schlüsselkomponenten gliedern:

a) Sprachkompetenz (Primär- und Sekundärenkulturation)

b) Kulturkompetenz (Bikulturalität)

c) Fachkompetenz

d) Recherchen- und Technikkompetenz

Alle vier Komponenten gemeinsam befähigen den Übersetzer dazu, unter Bezugnahme auf einen Ausgangstext über Kulturgrenzen hinweg ein adressatengebundenes Produkt in der Zielsprache herzustellen.

\section{Sprachenkompetenz - Enkulturation}

Die Qualität des translatorischen Handelns wird von der Kenntnis der Mutter- und der Fremdsprache geprägt. Die Beherrschung beider ist Voraussetzung für die Ausbildung zum Translator, was aber nicht bedeutet, dass sie im Rahmen des Studiums nicht weiter ausgebaut und gefestigt werden kann und muss.

Im Beitrag wurde mehrmals erwähnt, dass der Translator nicht nur die Sprachen beherrschen muss, mit denen er/sie arbeitet, sondern auch entsprechende kulturelle Kompetenzen aufweisen muss. Der Erwerb der Muttersprache ist nicht unbedingt gleichzusetzen mit dem Erwerb einer zweiten oder dritten Sprache bzw. Kultur. Vermeer bezeichnet die muttersprachi- 
ge Kompetenz als „Enkulturation“ und die fremdsprachige Kompetenz als „sekundäre Enkulturation" (vgl. /17/). Wird davon ausgegangen, dass das Fremde immer auf das Eigene bezogen wird und auf der Basis von bereits Vorhandenem interpretiert wird, dann ist auch das Wissen über eine fremde Kultur mit dem Wissen über eigene Kultur verbunden. Tritt man in Interaktion mit Partnern aus fremden Kulturen, wird unbewusst auf die eigenen Kommunikationsregeln zurückgegriffen, wobei erhebliche Divergenzen auftreten können in Form von Verständigungsschwierigkeiten. Daraus leitet Ammann ihre Definition der translatorischen Kulturkompetenz ab und versteht darunter „,... die Fähigkeit, von der eigenen Kultur und Situation abstrahieren zu können, die fremde Kultur in ihrer Besonderheit und im Vergleich $\mathrm{zu}$ der eigenen $\mathrm{zu}$ betrachten und die dabei gemachten Beobachtungen und Annahmen in einer bestimmten (kommunikativen) Situation ziel- und kulturgerecht anzuwenden." /18/

Am Anfang der translatorischen Ausbildung wird der Zusammenhang zwischen Kultur und Sprachverwendung daher am Beispiel der Muttersprache bewusst gemacht. Den nächsten Schritt der Auseinandersetzung mit der interkulturell geprägten Sprache stellt die kontrastive Paralleltextanalyse in der Mutter- und Fremdsprache dar, denn die angehenden TranslatorInnen werden damit konfrontiert, Texte zu übersetzen und zu dolmetschen. Der erste Schritt im Prozess der Translation ist es, so viel wie möglich zu verstehen, nicht nur einzelne Sätze, sondern den Text in seiner Gesamtheit. Manguel /19/ bezeichnet „das Übersetzen als die intensivste Form des Verstehens", was in enger Verbindung mit der Kompetenz steht, sich in andere hineinzuversetzen und nachzuempfinden was in einem Menschen vorgeht, wenn er etwas sagt. Als didaktisches Mittel werden auch Parelleltexte harangezogen, um den Studierenden zu zeigen, dass Textbausteine als konventionsbedingte Elemente miteinander verglichen werden müssen.

Da der Text im Mittelpunkt des translatorischen Handelns steht, ist das Hauptziel der TranslatorInnenausbildung die Entwicklung der übersetzungsrelevanten Textkompetenz. Diese besteht aus der Fähigkeit, Texte zu ver- stehen und zu produzieren /20/. Wie die Kompetenz "Verstehen" didaktisch umgesetzt werden kann ist nicht definitiv geklärt, es ist aber offensichtlich, dass Kommunikation wesentlich mehr ist als nur "Textarbeit" und, dass man im Translationsunterricht mehrere Disziplinen einbeziehen muss. „Um einen Text verstehen zu können, sind Semiotik und Hermeneutik unentbehrliche Wissenschaften, was uns an die Interdisziplinarität der Übersetzungswissenschaft und der Kulturstudien erinnert", behauptet Schwend /21/.

\section{Bikulturelle Kompetenz und funktionales Übersetzen}

Da der Übersetzer meist zwischen zwei Kulturkreisen vermittelt, ist das Bewusstmachen von kulturspezifischen Elementen der Ausgangs- und Zielsprachenkultur eines der Lernziele in der Ausbildung. Definiert man Kultur als Gesamtheit der Konventionen und Normen in einer bestimmten Gesellschaft, dann bedeutet Kulturkompetenz das Beherrschen dieser Konventionen und Normen. In der Mutterbzw. Primärkultur, in die man hineingewachsen ist, ist diese Kompetenz vorhanden. Man ist mit bestimmten Kulturelementen vertraut, auch ohne sich dessen stets bewusst zu sein. Beim Kontakt mit einer fremden bzw. sekundären Kultur wird deren Struktur nach den Maßstäben der eigenen Kultur interpretiert, meistens allerdings unbewusst, was für professionelle ÜbersetzerInnen nicht ausreichend ist. Translatorische Kompetenz erfordert Kompetenz in der eigenen sowie in der fremden Kultur (vgl. /22/, /23/).

Je nach Nähe oder Distanz der Kulturen gibt es graduelle Unterschiede. Wesentliche kulturelle Unterschiede sind schon zwischen Hessen, Schleswig-Holstein oder Sachsen auszumachen, geschweige denn zwischen West- und Ostdeutschland oder gar Deutschland und Slowenien; denn die Unterschiede sind natürlich mit zunehmender Entfernung weitaus größer, wie etwa zwischen Slowenien und Albanien oder zwischen europäischen und asiatischen Kulturen. Wenn wir Übersetzung als Vermittlung über kulturelle Grenzen hinweg verstehen, dann sind ÜbersetzerInnen transkulturell tätig - im 
Unterschied zur interkulturellen Kommunikation, bei der die Position der Vermittlerin/des Vermittlers wegfällt (vgl. /24/).

Dieses interkulturelle bzw. transkulturelle Grundwissen liefern den Studierenden außer den theoretischen Vorlesungen auch die im Unterricht eingesetzten Texte. Die Lehrkräfte zeigen nicht nur die innerhalb der Texte angesiedelten kulturellen Kontexte und Situationen auf, sondern machen die Studierenden auch auf die Verwendung von Textsorten, Registern und Sprachgebrauchsnormen in der jeweiligen Sprachkultur aufmerksam.

Hauptaufgabe des Translators ist es, die Information bzw. Mitteilung eines schriftlichen Textes $\mathrm{zu}$ verstehen und in einer anderen Sprache funktionsgemäß wiederzugeben.

Die funktionale Translationstheorie bezeichnet Translation als Sondersorte des kommunikativen Handels, welches kulturspezifisch ist. Man geht davon aus, dass Translation mit einem kulturellen Transfer gleichkommt. »Die Dominante aller Translation ist deren Zweck «, stellen Reiß/Vermeer /25/ fest, d.h. Texte werden immer für einen bestimmten Zweck geschaffen und Translation ist folglich immer zweckorientiert. TranslatorInnen können einen Text bzw. Fachtext kompetent übersetzen, wenn sie ihn zuvor verstanden haben, und Verstehen ist nur möglich, wenn schon ein gewisses Vorverständnis vom Inhalt vorhanden ist. Für die Produktion eines Translats ist es daher wichtig $\mathrm{zu}$ wissen, wer, wann, warum, für wen und wo diesen Ausgangstext produziert hat. Dabei muss als Erstes eine ganzheitliche Betrachtung des Textes erfolgen, d.h. der Text durchgelesen und in seinem außersprachlichen, kulturbedingten Kontext situiert werden. Viele Fragen werden schon vom Text selbst her beantwortet, der Titel bzw. die Überschrift beispielweise liefert schon einen relevanten Hinweis auf die Textsortensituierung und den Textinhalt.

Die erforderliche Translationskompetenz der ÜbersetzerInnen kann man als schichtartiges, modulähnliches System betrachten und besteht aus mehreren Teilkompetenzen:

1. sprachliche Kompetenz (Grammatik, Text, Soziolinguistik)
2. außersprachliche Kompetenz (Bikulturalität, themenbezogenes Wissen)

3. translatorische Kompetenz (Rezeption und Produktion)

4. professionelle Kompetenz (Technologie und Marktspezifikum)

Der Translator muss in der Lage sein, eine angemessene Übersetzungsstrategie zu finden, um dem Zweck des Textes in der Zielkultur gerecht $\mathrm{zu}$ werden und $\mathrm{zu}$ bestimmen, ob die Übersetzung überhaupt sinnvoll ist oder nicht. $\mathrm{Zu}$ vermitteln ist dem zielsprachigen Adressanten die ursprüngliche Information der Ausgangskultur. Reiß/Vermeer vertreten daher die Ansicht, dass eine Translation nur dann durchgeführt werden soll, »wenn dies nach den Umständen der Zielkultur (und - sprache) für den Translator (bzw. durch ihn für jemanden, 'der infrage kommt') angebracht erscheint « /26/. ÜbersetzerInnen müssen also über eine doppelte Kulturkompetenz verfügen: „,... eine bewusste Kompetenz in der Mutterkultur und in der Fremdenkultur(en) wird erst dann zur translatorischen Kulturkompetenz, wenn, darauf aufbauend, die bewusste Kompetenz zwischen Mutterkultur und Fremdkultur vorhanden ist" /27/. Zum interkulturellen Grundwissen können mit Löwe /28/ folgende Kategorien bzw. Fragestellungen gezählt werden:Individuum-Gesellschaft, IndividuumStaat, Parakultur-Diakultur-Idiokultur, kollektives Gedächtnis, Wertvorstellungen, Hierarchie, Autorität, Verhältnis der Generationen, der Geschlechter, öffentliches privates Verhalten, Körpersprache, Stellenwert kultureller Phänomene, Wohnen, Essen und Trinken, Arbeit, Freizeit, Selbstverständnis der Gesellschaft, Traditionen der Selbstdarstellung, Stereotype, Verhältnis zu anderen Gesellschaften, Tabus, Erkennen von Kulturgebundenheit, Gesetzmäßigkeiten des Kulturschocks. Der Translator, der zwischen Angehörigen verschiedener Kulturen professionell als Übersetzer, als Dolmetscher, als Regional- und Sprachexperte tätig sein will, muss also über diese doppelte Kulturkompetenz verfügen. 


\section{Fach-, Recherchen- und Technikkompe- tenz}

Übersetzer kommen bei ihrer Arbeit mit den unterschiedlichsten Themenbereichen und damit Fachsprachen in Kontakt. Fachübersetzen ist eine anspruchsvolle Tätigkeit, wobei der fachsprachige Übersetzer die Denkweise der jeweiligen Fachrichtung beherrscht und die Fähigkeit hat, Sachverhalte und fachliche $\mathrm{Zu}$ sammenhänge $\mathrm{zu}$ erkennen und sie richtig in der Zielsprache auszudrücken. Mit der fachsprachigen Übersetzung hängen viele translatorische Probleme zusammen und das Fachübersetzen ist nicht nur eine Substitution von fachlichen Ausdrücken. „Einig ist man sich darin, dass die Übersetzung fachsprachlicher Texte ebenso anspruchsvoll ist wie die literarische Übersetzung. Denn die laienhafte Vorstellung, bei Fachübersetzungen genüge im Wesentlichen die Substitution fachlicher Terminologie mit Hilfe eines grammatischen Minimums, ist erwiesenermaßen falsch.“ /29/ Den Studierenden wird in verschiedenen Gebieten eine grundlegende fachsprachige Kompetenz vermittelt, die sie später je nach Bedarf ausbauen und auf andere, von ihnen ausgewählte Bereiche ausweiten können, bis hin zum Experten. In der Fachkommunikation herrscht eine enge Beziehung zwischen dem Thema und seiner sprachlichen Umsetzung. Es werden exakte Begriffe verwendet, die möglichst genau übersetzt werden müssen. Die Fachleute, die auf diesem Gebiet kommunizieren, streben danach, diese Begriffe begrifflich-systematisch zu beschreiben und deren Sachverhalte zu präzisieren.

Die Sprache der Fachtexte, die ein Instrument zur Benennung der Gegenstände und Begriffe ist, orientiert sich an einer spezifischen Lexik, die in diesem Zusammenhang eine referentielle Funktion hat. Auf der fachsprachigen Ebene ist die Kreativität des Übersetzers stark eingeschränkt, da ein terminologischer Irrtum zu erheblichen Störungen im Kommunikationsprozess führen kann. Daher wird der Entwicklung der Recherchenkompetenz im Translationsunterricht einen hohen Stellenwert zugeteilt, um den eigenen Informations- und Dokumentationsbedarf ermitteln zu können und Strategien für die terminologische Arbeit zu entwickeln. Unterweisung in Computerarbeit ist dabei unumgänglich, denn ohne elektronische Hilfsmittel bzw. rechnergestützte Übersetzungshilfen (RGÜ) kommen Translatoren heute nicht mehr aus. Von daher betrachten wir die Schulung in Benutzung und Anwendung von Computerprogrammen, die eine wesentliche Unterstützung bei der translatorischen Tätigkeit leisten, auch als eines der Lernziele in der Ausbildung.

\section{Entwicklung der Dolmetschkompetenz}

Mit der Beherrschung von zwei oder mehreren Sprachen ist die Kompetenz zum Dolmetschen nicht gegeben. In den wissenschaftlichen Kreisen besteht ein Konsens darüber, dass Mehrsprachigkeit eine notwendige, nicht aber hinreichende Bedingung für die professionelle Ausübung des Dolmetschens darstellt. Das Kompetenzanforderungsmodell für Dolmetscher behauptet Pöchhacker besteht aus der „translatorischen Kompetenz, die auf Sprachund Kulturkompetenz aufbaut und vor allem auch den kognitiven und sprachlichen Umgang mit dem jeweiligen Wissensgebiet, Sachbereich oder Gegenstand der Kommunikation miteinschließt."/30/ Gut entwickelte Dolmetschkompetenzen gewährleisten dem Dolmetscher ein dem Dolmetschauftrag angemessenes Handeln unter Beachtung der zweckorientierten Vermittlung. Das Dolmetschen baut auf völlig automatisierten kognitiven Prozessen auf. In der Unterstützung der strategischen Vorgänge sieht man das Hauptziel in der Ausbildung der Dolmetscher.

Während in der Übersetzungsdidaktik vornehmlich das Übersetzungsprodukt im Mittelpunkt steht, muss die Dolmetschdidaktik in erster Linie auf dem Prozess aufbauen. Die Grundlage für den prozessorientierten Unterricht bilden somit die Vorgänge, die im Dolmetschprozess ablaufen, aber auch eine Qualitätskontrolle zur Sicherung der Qualität, wobei die Fähigkeit Äquivalenzbeziehungen zu schaffen in den Vordergrund steht. Teržan Kopecky stellt zu Recht fest: „Wenn man dolmetscht oder übersetzt werden in unserer Psyche Brücken zwischen zwei Sprachsystemen gebaut... und Brücken bauen, bedeutet $\ddot{\text { Aquiva- }}$ 
lenzbeziehungen suchen."/31/ Die Intention des Studiums ist verantwortungsvolle und selbstbewusste Dolmetscher als Kommunikationsprofis für den multikulturellen internationalen Markt auszubilden.

\section{Schlussbemerkung}

In der heutigen globalisierten und multilingualen Weltkommunikation ist die TranslatorInnenausbildung von bedeutender Rolle, besonders im Kontext der europäischen Union, die auf dem Prinzip von Mehrsprachigkeit und kultureller Vielfältigkeit basiert. Ptačnikova /32/ betont: „Es liegt auf der Hand, dass die Erweiterung der Europäischen Union die Notwendigkeit der internationalen Kommunikation noch verstärkte. Die politische Zusammenarbeit, der wissenschaftliche und kultureller Austausch, die Kooperation auf dem Gebiet der Technik und Wirtschaft und zahlreiche Geschäftskontakte, das alles trägt dazu bei, dass sprachliche und kulturelle Barrieren überwunden werden müssen, um allen mit diesem Prozess verbundenen Auflagen gerecht zu werden. Daraus ergeben sich erhöhte Anforderungen an die fremdsprachige Kompetenz aller Beteiligten." Translation muss als soziokognitives Handeln betrachtet werden, wobei man zukünftige TranslatorInnen für die historischen und aktuellen Aspekte der Translationswissenschaft sensibilisiert. Die Entwicklung der translatorischen Kompetenzen gehört zu den Hauptzielen des Translatorikstudiums an der Universität in Maribor.

Das Curriculum des BA/MA Studiumgangs wurde so entworfen, dass es mit entsprechenden anderen, anerkannten europäischen Hochschulprogrammen kompatibel ist.

\section{Vermerke}

/1/ DGT hat im April 2007 die EMT-Expertengruppe eingesetzt mit der Aufgabe, konkrete Vorschläge für den europäischen Master-Studiengang Übersetzen (European Master's in Translation - EMT) zu unterbreiten. Näheres zu diesem Projekt: http://ec.europa.eu/emt.

/2/ Koller, W., 2001, Einführung in die Übersetzungswissenschaft, UTB, Quelle \& Meyer, Wiebelsheim., S.59.
/3/ Im Bildungsbereich bezeichnet Portfolio eine $\mathrm{Zu}$ sammenstellung von Arbeiten und Dokumenten, die als Leistungspräsentation betrachtet werden.

/4/ Ammann, M., 1990, „Fachkraft oder Mädchen für alles? - Funktion und Rolle des Translators als Dolmetscher und Begleiter ausländischer Delegationen“, In: Vermeer, H.J. (ed.) Kulturspezifik des translatorischen Handelns, 2. Auflage, Heidelberg, S.83.

/5/Translation wird als Oberbegriff für Übersetzen und Dolmetschen gebraucht.

/6/ Prunč, E., 2001, Einführung in die Translationswissenschaft, Institut für Translationswissenschaft, Graz.

/7/ Malgorzewicz, A., Entwicklung der translatorischen Kompetenz im Rahmen des Postgradualen Studiums für Sprachmittler an der Universität Wroclaw, http://www.fask.unimainz.de/user/hagemann/publ/malgozewicz.pdf (12.3.2008)

/8/ Vermeer, H.J., 1986, Übersetzen als kultureller Transfer, In: Snell-Hornby (ed.), Übersetzungswissenschaft. Eine Neuorientierung. 2. Aufl. Tübingen, Basel, Francke Verlag, S.43.

/9/ Risku, H., 1999, „Kognitionswissenschaft“, In: Handbuch Translation, Hrsg. M. Snell-Hornby, H. G. Hönig, P. Kußmaul, P. A. Schmitt, Tübingen, $\mathrm{S} .111$.

/10/ Koller, W., 2001, Einführung in die Übersetzungswissenschaft, UTB, Quelle \& Meyer, Wiebelsheim., S.232.

/11/ Ibid

/12/Reiß, K., Vermeer, H.J., 1991, Grundlegung einer allgemeinen Translationstheorie, Tübingen, Niemayer, 2. Auflage., S.4

/13/ Stolze, R., 2001, Übersetzungstheorien. Eine Einführung, Tübingen, Narr Verlag, S.178.

/14/ Vgl. Holz-Mantääri, J.: Translatorisches Handeln - Theoretisch fundierte Berufsprofile. In: SnellHornby, M. (Hrsg.): Übersetzungswissenschaft. Eine Neuorientierung, UTB 1986

/15/ Hönig, H. G./Kußmaul, P.: Strategie des Übersetzens. Ein Lehr- und Arbeitsbuch. Tübingen, 1984

/16/ Vermeer, H./Reiß, K.: Grundlegung einer allgemeinen Übersetzungswissenschaft, 1991

/17/ Vermeer, H.J., 1986, Übersetzen als kultureller Transfer, In: Snell-Hornby (ed.), Übersetzungswissenschaft. Eine Neuorientierung. 2. Aufl. Tübingen, Basel, Francke Verlag

/18/ Ammann, M., 1990, „Fachkraft oder Mädchen für alles? - Funktion und Rolle des Translators als Dolmetscher und Begleiter ausländischer Delegationen“, In: Vermeer, H.J. (ed.) Kulturspezifik des translatorischen Handelns, 2. Auflage, Heidelberg, S.71 
/19/ Manguel, A., 1999, Eine Geschichte des Lesens, Reinbek bei Hamburg, Rowohlt Taschenbuch Verlag., S.310

/20/ Best, J., 2002, „Die Bedeutung der grundsprachlichen Kompetenz in der Übersetzer- und Dolmetscherausbildung“, In: Übersetzen und Dolmetschen: Eine Orientierungshilfe, Hrsg. J. Best, S. Kalina, Tübingen, S. 126.

/21/ Schwend, J., 1997, „Kultur, Kulturwissenschaft und Translation“, In: Drescher, H.W.(ed.) Transfer: Übersetzen -Dolmetschen-Interkulturalität, Frankfurt/M./Berlin/Bern/ New York/Paris/Wien: Peter Lang (FASK Publikationen des Fachbereichs Angewandte Sprach- und Kulturwissenschaft der Johannes Gutenberg Universität Mainz in Germersheim, Reihe A, Bd.23), S. 272.

/22/ Göhring, H. 1978, „Interkulturelle Kommunikation: Die Überwindung der Trennung von Fremdsprachen- und Landeskundenunterricht durch einen integrierten Fremdverhaltensunterricht“", In: Hartig,M., Wode, H. (eds.) Kongressberichte der 8. Jahrestagung der Gesellschaft für Angewandte Linguistik, Stuttgart, S. 10.

/23/ Witte, H., 1987, „Die Kulturkompetenz des Translators - Theoretische-abstrakter Begriff oder realisierbares Konzept?“, In: TextconText, 2, 2/3, S. 109.

/24/ Prunč, E., 2001, Einführung in die Translationswissenschaft, Institut für Translationswissenschaft, Graz., S.108
/25/ Reiß, K., Vermeer, H.J., 1991, Grundlegung einer allgemeinen Translationstheorie, Tübingen, Niemayer, 2. Auflage., S.96

/26/ Reiß, K., Vermeer, H.J., 1991, Grundlegung einer allgemeinen Translationstheorie, Tübingen, Niemayer, 2. Auflage., S.84

/27/ Löwe, B., 2002, „Translatorische Kulturkompetenz: Inhalte - Erwerb - Besonderheiten", In: Übersetzen und Dolmetschen: Eine Orientierungshilfe, Hrsg. J. Best, S. Kalina, Tübingen, S. 150.

/28/ Löwe, B., 2002, „Translatorische Kulturkompetenz: Inhalte - Erwerb - Besonderheiten", In: Übersetzen und Dolmetschen: Eine Orientierungshilfe, Hrsg. J. Best, S. Kalina, Tübingen, S. 151.

/29/ Fluck,H.R., 1998, Fachsprachen. Einführung und Bibliographie, Tübingen/Basel., S.136.

/30/ Pöchhacker, F., 1998, Dolmetschen. Konzeptuelle Grundlagen und deskriptive Untersuchungen, Wien, Habilschrift., S.44.

/31/ Teržan-Kopecky, K., 2001, Psihološke dimenzije jezikovnih ravnanj, Univerza v Mariboru., S.125.

/32/ Ptačnikova,V., 2005, „Fachübersetzen im Kontext der europäischen Integration“, In: Informatologia, 38, Croatian Communication Association, Zagreb, S.25

/33/ Kučiš, V., 2008, Competence development for translators and interpreters - the case of the department for translation science at the University of Maribor, Informatologia(41), No.2, 149-155. 


\title{
COMPETENCE DEVELOPMENT FOR TRANSLATORS AND INTERPRETERS - THE CASE OF THE DEPARTMENT FOR TRANSLATION SCIENCE AT THE UNIVERSITY OF MARIBOR
}

\author{
Vlasta Kučiš \\ Department of Translation Science, Faculty of Philosophy, University of Maribor, Maribor, Slovenia
}

\begin{abstract}
This paper is a first attempt at making an analysis and trying to present a summary of the main characteristics of a newly founded undergraduate program for Translators and Interpreters at the Faculty of Philosophy, University of Maribor. The author tried to outline the structure of the program, which is based on the development of competences in the field of translation science, with the aid of theoretical and didactic approaches. The policy of EUintegration, demands qualified translators and interpreters, and the profession of translation aspires to unified educational criteria. Theoretical and scientific postulates confirm the presented strategy of education in the context of harmonization and optimizing European undergraduate programs and curriculums for future translators and interpreters.
\end{abstract}

Keywords

translation science; education programme; translator 